\title{
Local Structure of Nanopowders of Refractory Nitrides Used to Increase the Critical Current of High-Temperature Superconductors
}

\author{
A. P. Menushenkov ${ }^{a}$, I. A. Rudnev ${ }^{a}$, I. A. Garifullin ${ }^{b}$, R. V. Chernikov ${ }^{a}$, \\ O. V. Kashurnikova ${ }^{a}$, and B. P. Mikhailov ${ }^{c}$ \\ ${ }^{a}$ Moscow Engineering Physics Institute, Kashirskoe sh. 31, Moscow, 115409 Russia \\ e-mail:menushen@htsc.mephi.ru \\ ${ }^{b}$ Zavoisky Physical-Technical Institute, Kazan Scientific Center, Russian Academy of Sciences, \\ Sibirskii trakt 10/7, Kazan, 420029 Russia \\ ${ }^{c}$ Baikov Institute of Metallurgy and Materials Science, Russian Academy of Sciences, \\ Leninskii pr. 49, Moscow, 119991 Russia
}

\begin{abstract}
The effect of dispersivity on the local-structure features in $\mathrm{ZrN}$ nanocrystals has been investigated by X-ray absorption (EXAFS) spectroscopy. The Debye-Waller factors and bond lengths have been determined for $\mathrm{Zr}-\mathrm{N}$ and $\mathrm{Zr}-\mathrm{Zr}$ coordination spheres. It is found that a decrease in the nanoparticle size leads to an increase in the static Debye-Waller factor for the $\mathrm{Zr}-\mathrm{Zr}$ bond. The scale of changes is comparable with the increase in the Debye-Waller factor upon heating from 15 to $77 \mathrm{~K}$.
\end{abstract}

DOI: $10.3103 / \mathrm{S} 1062873808080121$

Application of high-temperature superconducting (HTSC) materials in power engineering, transport, and industrial physics implies fabrication of long winding conductors on their basis. Such conductors should primarily have a high critical current density $J_{c}$, which is provided by the presence of natural or artificial effective centers of magnetic flux pinning in a material.

A technologically convenient methods for fabricating additional pinning centers and, therefore, improving the transport characteristics of HTSC materials is the incorporation introduction of nanoscale inorganic additives (inert with respect to the superconducting matrix) into their volume in the synthesis stage. Such impurities should be chosen so that they, on the one hand, would not decrease the critical temperature of the initial superconductor and, on the other hand, play the role of effective pinning centers when incorporated introduced into a superconducting material. Investigations on introduction of nanoadditives of magnesium oxide; niobium, tantalum, titanium, and silicon carbides; and hafnium nitride into HTSC materials have been performed [1]. The results obtained showed that the critical current density in HTSC compounds $(\mathrm{Bi}, \mathrm{Pb})_{2} \mathrm{Sr}_{2} \mathrm{CaCu}_{2} \mathrm{O}_{8+x} \quad(\mathrm{Bi} 2212) \quad$ and $(\mathrm{Bi}, \mathrm{Pb})_{2} \mathrm{Sr}_{2} \mathrm{Ca}_{2} \mathrm{Cu}_{3} \mathrm{O}_{10+x}(\mathrm{Bi} 2223)$ can be increased [1,2].

One of promising compounds for doping HTSC materials is ultradispersed $\mathrm{ZrN}$ powder [3]. For example, at a $\mathrm{Zr}$ concentration of $0.27 \mathrm{wt} \%$ powder, the critical current in Bi2223 increased by a factor of a more than twofold increase in the critical current in Bi2223 was found (Fig. 1). At the same time, the question about the optimal average size (dispersivity) of introduced impurity particles remains open. For example, investigation of the dependence of the critical current on the dispersivity of introduced $\mathrm{Si}_{3} \mathrm{~N}_{4}$ and $\mathrm{ZrN}$ nanoadditives showed that there is some average size corresponding to the at which the maximum increase in the critical current is maximum.

To get insight into the physical aspects of interaction of ultradispersed powders with the HTSC matrix, we investigated the dependences of the local structure

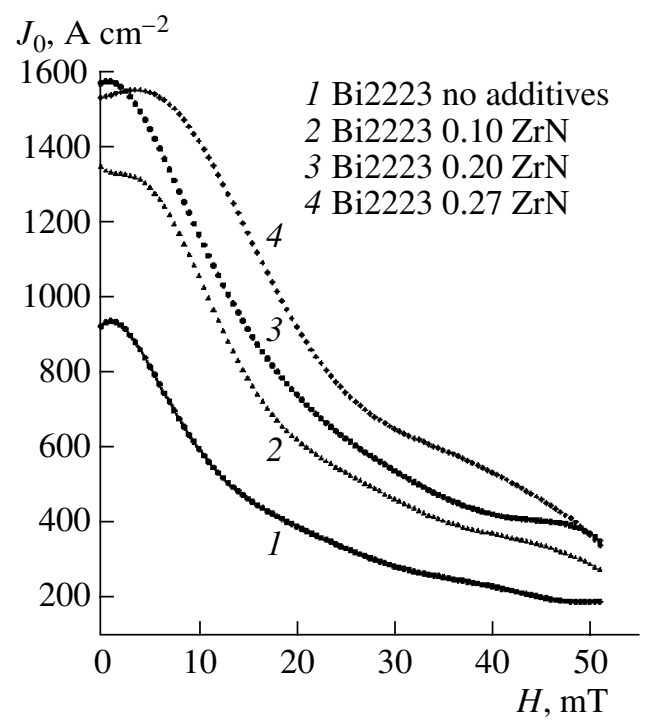

Fig. 1. Dependences of the critical current for $\mathrm{Bi} 2223$ on the grain size of incorporated $\mathrm{ZrN}$ nanoadditives at their different mass concentrations. 
parameters of zirconium nitride on the grain size by the extended X-ray absorption fine structure (EXAFS) spectroscopy.

$\mathrm{ZrN}$ nanopowders were obtained by successive grinding of an initial crystalline sample (with an average grown size of $4.22 \mu \mathrm{m})$ in a ball mill to the ultradispersed state. The average grain size after double and qquadruple grinding was $0.56 \mu \mathrm{m}$ and $0.47 \mu \mathrm{m}$, respectively.

The EXAFS spectra above the $\mathrm{Zr} K$-absorption edge were measured in the $A 1$ line at HASYLAB (DESY, Hamburg) at temperatures of 15,77 , and $300 \mathrm{~K}$, using a pumped liquid-helium cryostat. Treatment and simulation of the EXAFS spectra were performed using the VIPER program [4], according to the standard formula

$$
\begin{gathered}
\chi(k)=-S_{0}^{2} \sum_{n} \frac{1}{k R_{n}^{2}} N_{n}\left|f_{n}(\pi, k)\right| \\
\times \exp \left(-2 k^{2} \sigma_{n}^{2}\right) \exp \left(2 k R_{n}+2 \delta_{1}(k)+\phi_{n}(\pi, k)\right),
\end{gathered}
$$

where $\chi(k)$ is the EXAFS function, $N_{n}$ is the coordination number, $R_{n}$ is the average radius of the $n$th coordination sphere, and $\sigma_{n}^{2}$ is the root-mean-square deviation of the interatomic distance from its mean, referred to as the Debye-Waller factor. In $X$-ray absorption spectroscopy, the Debye-Waller factor $\sigma^{2}$ is the sum of the temperature-independent contribution $\sigma_{s}^{2}$, characterizing the static disordering of atoms, and the dynamic contribution $\sigma_{d}^{2}$, which characterizes the change in the interatomic distance due to thermal vibrations. The scale factor $S_{0}^{2}$ takes into account manybody effects. The backscattering amplitudes $f_{n}(\pi, k)$ and phases of $2 \delta_{1}(\pi, k)+\phi_{n}(\pi, k)$ were calculated using the FEFF 8.20 program [5] on the basis of the diffraction data. The simulation made it possible to determine the Debye-Waller factors and $\mathrm{Zr}-\mathrm{N}$ and $\mathrm{Zr}-\mathrm{Zr}$ bond lengths.

Figure 2 shows the EXAFS functions $\chi(k) k^{2}$, measured above the $\mathrm{Zr} K$-absorption edge at $T=77 \mathrm{~K}$ for the initial crystalline $\mathrm{ZrN}$ sample and the samples subjected to double and qudruple grinding and the Fourier transform moduli of these functions. A decrease in the amplitudes of the maxima corresponding to the zirconium coordination spheres is well pronounced. Such behavior may indicate an increase in the local structure disordering, occurring throughout the entire crystallite bulk.

The results of numerical simulation of the local structure parameters (see the table) show that an increase in the $\mathrm{ZrN}$ dispersivity increases the static Debye-Waller factor of the $\mathrm{Zr}-\mathrm{Zr}$ bond by a value comparable with the dynamic Debye-Waller factor at room temperature (Fig. 3). According to the data obtained, the degree of disorder in the $\mathrm{Zr}$ sublattice is $\sim 0.02 \AA$ and $\sim 0.04 \AA$ for double and qudruple grinding of the initial sample, respectively.
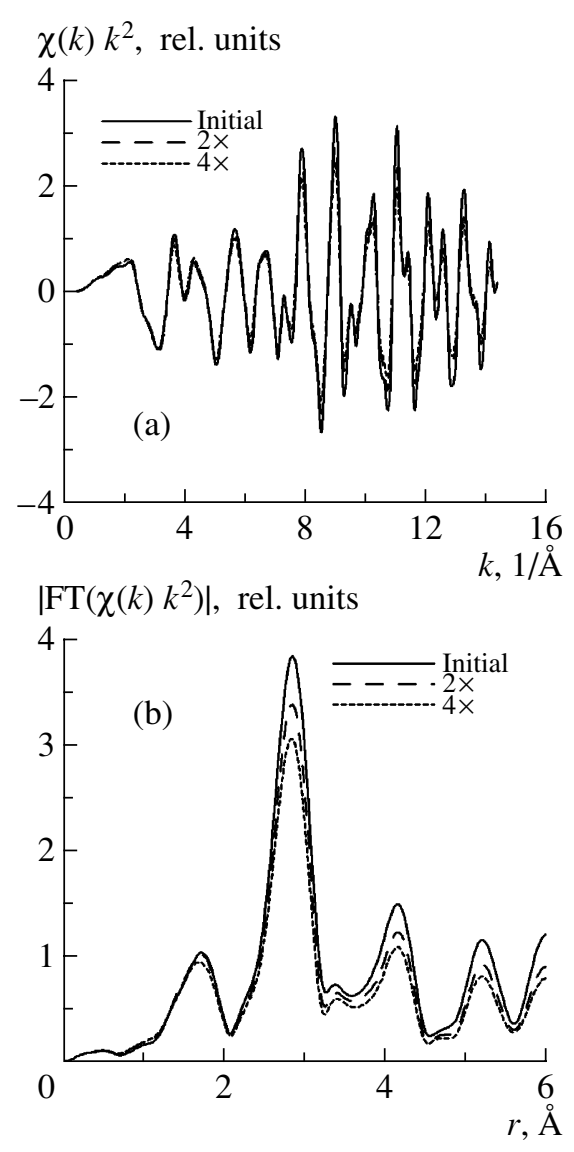

Fig. 2. (a) EXAFS functions $\chi(k) k^{2}$, measured above the $\mathrm{Zr}$ $K$-absorption edge at $T=77 \mathrm{~K}$, and (b) their Fourier transform moduli.

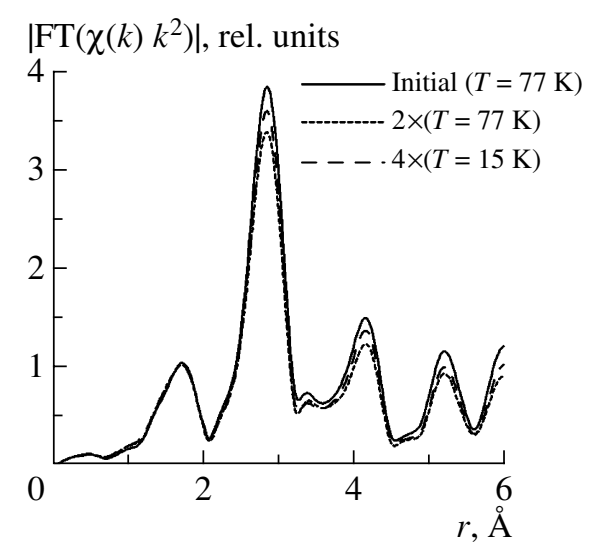

Fig. 3. Effect of the particle size $r$ and temperature on the local crystal structure of $\mathrm{ZrN}$ nanopowders.

Thus, it has been shown that a change in the size of nanoparticles affects the parameters properties of their local crystal structure. Furthermore, we plan to perform a complex investigation of the local structure of nanopowders incorporated directly into a superconducting matrix. Comparison of the local structural features of the nanoadditives incorporated into a matrix with the 
Parameters of the local crystal structure of $\mathrm{ZrN}$ nanopowders

\begin{tabular}{l|l|l|l|l|l|l}
\hline \multirow{2}{*}{} & \multicolumn{2}{|c|}{ Initial sample } & \multicolumn{2}{c|}{$D=560 \mathrm{~nm}$} & \multicolumn{2}{c}{$D=470 \mathrm{~nm}$} \\
\cline { 2 - 6 } & $T=15 \mathrm{~K}$ & $T=77 \mathrm{~K}$ & $T=15 \mathrm{~K}$ & $T=77 \mathrm{~K}$ & $T=15 \mathrm{~K}$ & $T=77 \mathrm{~K}$ \\
\hline$R, \AA(\mathrm{Zr}-\mathrm{N})$ & $2.273(8)$ & $2.275(9)$ & $2.27(1)$ & $2.27(1)$ & $2.26(1)$ & $2.26(1)$ \\
$\sigma^{2}, \AA^{2}(\mathrm{Zr}-\mathrm{N})$ & $0.0055(1)$ & $0.0061(1)$ & $0.0066(1)$ & $0.0064(1)$ & $0.0073(1)$ & $0.0076(2)$ \\
$R, \AA(\mathrm{Zr}-\mathrm{Zr})$ & $3.240(2)$ & $3.242(2)$ & $3.239(2)$ & $3.241(3)$ & $3.239(3)$ & $3.240(3)$ \\
$\sigma^{2}, \AA^{2}(\mathrm{Zr}-\mathrm{Zr})$ & $0.0028(1)$ & $0.0033(1)$ & $0.0037(1)$ & $0.0041(1)$ & $0.0041(1)$ & $0.0046(2)$ \\
\hline
\end{tabular}

structure of initial nanopowders makes it possible to determine the state of nanoadditives in the matrix, for example, absence or presence of impurity dissolution in the matrix, etc. Eventually, this approach will allow to explain the existence of the optimal size and concentration of nanoadditives for obtaining a maximum increase in the critical current.

\section{ACKNOWLEDGMENTS}

This study was supported by the Russian Foundation for Basic Research, project nos. 08-02-00759, 0603-32720, and 06-08-00551.

\section{REFERENCES}

1. Mikhailov, B.P., Rudnev, I.A., and Bobin, P.V., Inorg. Mater., 2004, vol. 40, no. Suppl. 2, p. 91.

2. Rudnev, I.A., Mikhailov, B.P., and Bobin, P.V., Pis'ma Zh. Tekh. Fiz., 2005, vol. 31, no. 4, p. 88.

3. Mikhailov, B.P., Rudnev, I.A., Bobin, P.V., et al., Pis'ma Zh. Tekh. Fiz., 2006, vol. 32, no. 20, p. 70.

4. Klementev, K.V., J. Phys. D: Appl. Phys., 2001, vol. 34, p. 209.

5. Rehr, J.J., Mustre de Leon, J., Zabinsky, S.I., et al., J. Am. Chem. Soc., 1991, vol. 113, p. 5135. 